\title{
Use of technology to facilitate a prospective surveillance program for breast cancer-related lymphedema at the Massachusetts General Hospital
}

\author{
Lauren M. Havens ${ }^{1}$, Cheryl L. Brunelle ${ }^{2}$, Tessa C. Gillespie ${ }^{1}$, Madison Bernstein ${ }^{1}$, Loryn K. Bucci ${ }^{1}$, \\ Yara W. Kassamani ${ }^{1}$, Alphonse G. Taghian ${ }^{1}$ \\ ${ }^{1}$ Department of Radiation Oncology, ${ }^{2}$ Department of Physical and Occupational Therapy, Massachusetts General Hospital, Boston, MA, USA \\ Contributions: (I) Conception and design: All authors; (II) Administrative support: None; (III) Provision of study materials or patients: None; (IV) \\ Collection and assembly of data: None; (V) Data anzalysis and interpretation: None; (VI) Manuscript writing: All authors; (VII) Final approval of \\ manuscript: All authors. \\ Correspondence to: Dr. Alphonse G. Taghian. Department of Radiation Oncology, Massachusetts General Hospital, Boston, MA, USA. \\ Email: ataghian@mgh.harvard.edu.
}

\begin{abstract}
Breast cancer-related lymphedema (BCRL) is a negative sequela of breast cancer (BC) caused by trauma to the lymphatic system during surgery or radiation to the axillary lymph nodes. BCRL affects approximately one in five patients treated for BC, and patients are at a lifelong risk for BCRL after treatment. Early diagnosis of BCRL may prevent its progression and reduce negative effects on quality of life, necessitating comprehensive prospective screening. This paper provides an overview of technology that may be used as part of a BCRL screening program, including objective measures such as perometry, bioimpedance spectroscopy, tissue tonometry, and three-dimensional optical imaging. Furthermore, this paper comprehensively reviews the technology incorporated into the established prospective screening program at Massachusetts General Hospital. Our prospective screening program consists of longitudinal measurements via perometry, symptoms assessment, and clinical examination by a certified lymphedema therapist (CLT) as needed. Discussion about use of perometry within the screening program and incorporation of arm volume measurements into equations to determine change over time and accurate diagnosis is included [relative volume change (RVC) and weight-adjusted change (WAC) equations]. Use of technology throughout the program is discussed, including a HIPPA-compliant online research database, the patient's electronic medical record, and incorporation of BCRL-related symptoms [BC and lymphedema symptom experience index (BCLE-SEI) survey]. Ultimately, both subjective and objective data are used to inform BCRL diagnosis and treatment by the CLT. In conclusion, the role of technology in facilitating BCRL screening is indispensable, and the continued development of objective assessment methods that are not only reliable and valid, but also cost-effective and portable will help establish BCRL screening as the standard of care for patients treated for BC.
\end{abstract}

Keywords: Lymphedema; breast cancer; prospective surveillance; screening; technology

Received: 23 October 2019. Accepted: 29 April 2020; Published: 20 January 2021.

doi: $10.21037 /$ mhealth-19-218

View this article at: http://dx.doi.org/10.21037/mhealth-19-218

\section{Introduction}

From 1989 to 2016, breast cancer (BC) mortality has decreased 40\%; therefore, patients are living longer after treatment (1). Furthermore, one in five patients treated for BC will develop BC-related lymphedema (BCRL), a result of $\mathrm{BC}$ treatment (2). BCRL is a negative sequela of $\mathrm{BC}$ caused by trauma to the lymphatic system by surgery or radiation to regional lymph nodes, which may impair 
lymphatic drainage (3). This mechanical insufficiency of the impaired lymphatic system results in the collection of cellular and proteinaceous lymphatic fluid in the interstitium, which may cause swelling in the affected limb (4). Previous studies have established risk factors including body mass index (BMI) $\geq 30 \mathrm{~kg} / \mathrm{m}^{2}$ at the time of $\mathrm{BC}$ diagnosis, low volume swelling, axillary lymph node dissection (ALND), regional lymph node radiation (RLNR), and cellulitis $(5,6)$. Additionally, a greater number of lymph nodes removed and nodes positive for metastatic disease increase risk of BCRL, although this may be a conduit for more intensive treatment of the lymph nodes and disease stage (6-11). Moreover, there are new insights into other potential risk factors for BCRL. Specifically, immediate reconstruction after mastectomy appears to decrease BCRL risk (12-16). Neoadjuvant (17) and taxanebased chemotherapy (18-20) regimens have been implicated to increase BCRL risk, although this remains somewhat controversial as other studies have not found a significant association between taxane-based chemotherapy and BCRL risk (21). Although a trend toward significance was found between trastuzumab treatment and breast lymphedema, this was a small study and further research is required (22). Association between tumor-specific features, such as peritumoral lymph-vascular invasion and extranodal extension and BCRL has also been found (20). Finally, there is preliminary evidence suggesting a possible role of genetic predisposition in BCRL development after BC $(23,24)$.

BCRL is a progressive and chronic illness requiring lifelong care and support, which makes early diagnosis crucial for its management. Severity of BCRL ranges, and it can be characterized into four stages (Ia-III): latent or subclinical lymphedema (changes in tissue composition and development of symptoms), spontaneously reversible (pitting edema), spontaneously irreversible (non-pitting edema), and lymphostatic elephantiasis (accumulation of adipose and fibrotic tissue) (5). Not only does BCRL cause a myriad of symptoms and functional impairments, it also significantly and adversely affects quality of life (QOL) $(5,25)$.

Detecting lymphedema through surveillance allows for early recognition, diagnosis, and treatment. This is preferable to an impairment-based model, wherein patients are diagnosed once clinical swelling is visible by examination and symptoms are apparent, because BCRL becomes less responsive to treatment and is associated with greater risk of complications and decreased QOL (26-31). A comprehensive prospective screening program is currently recommended by the International Society of Lymphology
(ISL), National Lymphedema Network (NLN), American Society of Breast Surgeons (ASBrS) and the National Comprehensive Cancer Network (NCCN) (5,32-37).

Since its establishment in 2005, the BCRL prospective screening program at Massachusetts General Hospital (MGH) has screened over 5,000 women $(5,32,37,38)$. We incorporate technology into clinic workflow to efficiently maximize patient care, to view screening data longitudinally throughout survivorship, and to facilitate communication within our multidisciplinary medical team. Technology also facilitates the capture of objective data electronically within a large research database, for ease of data organization, storage, and analysis. This paper will explore the role of technology in the MGH screening program and, more generally, the vital role of technology in facilitating the prospective detection of BCRL.

\section{Objective measurement of BCRL}

In their 2011 Position Statement, the NLN asserts that it is imperative to screen patients at risk of developing lymphedema, which is concordant with evolving evidence that early intervention may prevent the progression to irreversible BCRL (34,35,39-46). Multiple forms of measurement exist to detect BCRL. However, these methods are not interchangeable and variations in measurement of BCRL have made it difficult to standardize reported rates of BCRL (47-49). Regardless of method, measurements must be taken preoperatively to establish a baseline measurement and avoid misdiagnosis $(5,32,37,42,50)$. In a preclinical study performed using perometry, $28.3 \%$ of patients had a pre-existing arm asymmetry of $\geq 5 \%$, whereas $2.9 \%$ of patients had a pre-existing arm asymmetry of $\geq 10 \%$ (51). Furthermore, without a preoperative baseline measurement, $41.6 \%$ of patients were under diagnosed and $40.1 \%$ over diagnosed with BCRL at a relative volume change (RVC) $\geq 5 \%$ as compared to $50.0 \%$ and $54.8 \%$ of patients at RVC $\geq 10 \%$ (51). The NLN, ISL, ASBrS, NCCN, and the National Accreditation Program for Breast Centers (NAPBC) all recommend baseline measurements for accurate diagnosis $(5,32,37,50,51)$. In the following section, we will outline technology that facilitates the reliable, valid, and accurate diagnosis of BCRL.

\section{Perometry}

Infrared optoelectronic perometry is a reliable, valid, and accurate tool used in volumetric analysis for BCRL 


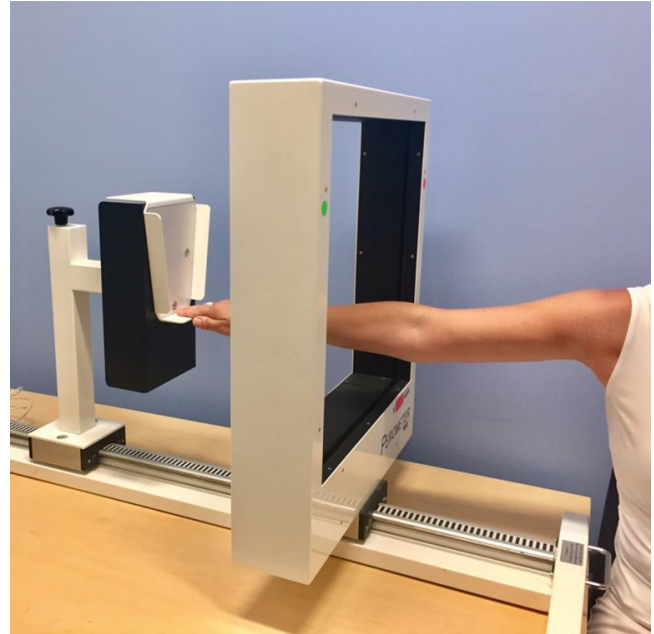

Figure 1 Perometer.

screening $(35,52,53)$. The Perometer (Figure 1) consists of a frame of infrared emitter and receiver pairs that slides along a track over the entire length of the limb, measuring from $53 \mathrm{~mm}$ from the fingertips to the axillary crease. The Perometer collects two-dimensional information from each arm to calculate arm circumference measurements every $4.7 \mathrm{~mm}$ and summates the volume using the truncated cone method (54). Measurements, collected in milliliters, are maintained within the PeroPlus $2000^{\circledR}$ software on a PC desktop (Figure 2) (55). To ensure the quality of Perometer measurements, one must confirm the arm is abducted to 90 degrees at the shoulder, fully flat and without bends in the elbow or wrist, making adjustments to the patient's position as needed (Figure 2). Although the Perometer is limited to measuring limb edema, immobile, and expensive $(33,54,56)$, it is reliable, valid, efficient, diagnostically accurate, able to detect subclinical BCRL, and requires a small space for measurement $(3,35,52,53)$. The Lymphedema Research Program at MGH incorporated perometry into its screening program in 2005 for the aforementioned reasons and its ability to maintain longitudinal clinical and research databases. The Perometer fits well into our clinic workflow and has become a mainstay of our screening program (3).

It should be noted that in the absence of perometry, limb volume may be calculated automatically using circumferential tape measurement (CTM). Circumferential girth measures taken at regular intervals along the arm (e.g., every 4 or $10 \mathrm{~cm}$ ) may be entered into commercially available software for volume calculation (57). With careful training and practice, CTM is a reliable and valid measurement of limb volume (49). Of note, limb volumes should incorporate volume at preoperative baseline and take into account the opposite arm as a comparison or body weight fluctuations, which will be further discussed.

\section{Bioelectrical impedance spectroscopy (BIS)}

BIS utilizes a low frequency current to measure impedance, affected by the opposition of extracellular fluid and body fluid (resistance and reactance, respectively) to electrical flow (58). Although it requires a small space for measurement and it is expensive, BIS has been shown to be a reliable and diagnostically accurate tool to diagnose and detect established BCRL (56,58-60). However, its ability to detect advanced lymphedema is limited as this is fat-, rather than fluid-dominant, and its ability to detect subclinical lymphedema is not strongly supported (61-63). Resistance and reactance are calculated over a range of frequencies to generate a Lymphedema Index (L-Dex) ratio (58). For unilaterally affected patients, the L-Dex score is a measure of the extracellular fluid in the affected limb (58). Previously, a patient was considered to have clinical lymphedema when the measured L-Dex score was $\geq+10$ units from baseline (40), the threshold for diagnosing clinical lymphedema has since been lowered to an L-Dex score $\geq+7.1$ units from baseline (64-66). Recently, it is thought that the threshold for diagnosis may be even lower, at a change in L-Dex $\geq+6.5$ units from baseline (65).

The L-Dex $\mathrm{U}_{400}{ }^{\circledast}$ has been utilized in the MGH BCRL screening program for research purposes, but has been discontinued by the manufacturer given the advent of newer BIS equipment, $\mathrm{SOZO}^{\circledR}$ (Figure $3 A$ ), which our team has since added in a research capacity (67). To obtain an L-Dex score using $\mathrm{SOZO}^{\circledast}$, the patient's palms and feet are aligned flat in the center of the SOZOtouch ${ }^{\circledR}$ and SOZOstep ${ }^{\circledR}$ plates, respectively (68). $\mathrm{SOZO}^{\circledR}$ performs impedance measurements at 256 frequencies (69), thereby generating a semicircular curve to extrapolate impedance values at R0 and Rinfinity (58), which are stored within the SOZOhub ${ }^{\circledR}$ online database (68). BIS measurements provide Cole plots as visual representation of the data output. Measured impedance values are plotted along a semicircular curve and experience varying degrees of scatter at the extreme ends of the range of resistance, reflective of interference in the measurement (Figure 3B) (69). Cole plots, thereby, should be used to assess the quality of the measurement (69). At $\mathrm{MGH}, \mathrm{SOZO}^{\circledR}$ is currently used solely for research to identify potential complementary roles of BIS with 

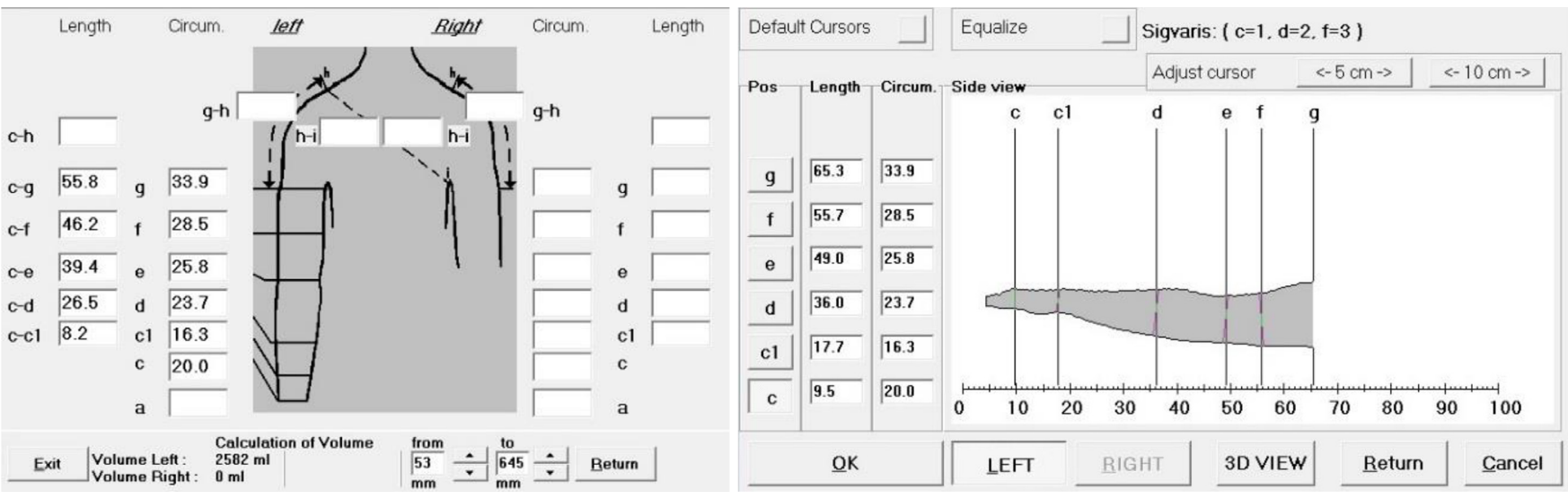

Figure 2 Perometer output within the PeroPlus $2000^{\circledR}$ software.

perometry. It is not currently used within our clinical screening program as its role has not been clearly delineated in terms of diagnosing subclinical BCRL, although other facilities do screen with $\mathrm{SOZO}^{\circledR}$ at this time.

\section{Tissue tonometry}

Tissue tonometry analyzes tissue water of the skin and cutis by measuring a tissue dielectric constant (TDC) at a frequency of $300 \mathrm{MHz}$ (54). The MoistureMeter- $\mathrm{D}^{\circledR}$ (Delphin Technologies) consists of a measuring unit and four handheld probes providing varying effective measurement depths $(0.5-5 \mathrm{~mm})$ (Figure 4). The appropriate probe is placed on the skin's surface for up to 10 seconds to measure a TDC, which increases proportionally with water content and edema (56). The TDC values may be converted to absolute local tissue water (LTW) values (\%), which have been found to be significantly different between patients with and without BCRL (71). In patients with BCRL, TDC values of the unaffected (contralateral) arm are not significantly different from TDC values measured in patients treated for BC without BCRL, or in healthy women (72). Studies have found that TDC values in the lymphedematous arm are significantly higher (44\% to $65 \%$ ) than those in the unaffected (contralateral) arm. (73,74).

TDC has an interclass correlation coefficient (ICC) of 0.996 (95\% CI: $0.965-1.000)$ for inter-rater reliability, and an ICC of 0.996 (95\% CI: 0.965-1.000) for intrarater reliability (54). Of note, trunk and breast edema affect approximately a third of patients treated for BC one year postoperatively $(58,59)$. As TDC may be used in any anatomic location, it may be helpful in identifying and quantifying edema in the breast and trunk, for example, for which there are limited measurement options $(60,61)$. Though not currently in use by the Lymphedema Research Program at MGH, we hope to explore tissue tonometry's ability to measure trunk and breast edema. Although expensive, tissue tonometry is sensitive, portable, can provide segmental and unilateral measurements, and can quantify edema in areas previously difficult to measure $(20,24,56,62)$. Its role in lymphedema screening and its ability to identify subclinical lymphedema continues to evolve in the literature.

\section{Three-dimensional (3D) optical imaging}

$3 \mathrm{D}$ depth-sensing imaging has been established as a novel tool to screen for BCRL (75-78). One method of 3D imaging, stereophotogrammetry, uses a 5 -pod setup of 15 cameras to capture visual information from the entire arm, capturing the lower and upper arm separately (75). In a 2016 study by Hameeteman et al., 3D stereophotogrammetry was confirmed to be a diagnostically valid and reliable method of screening for BCRL [ICC: 0.997 (95\% CI: 0.992-0.999), $\mathrm{P}<0.001)$ (75). In a follow-up study by Hoevenaren et al., $3 \mathrm{D}$ stereophotogrammetry was validated as an accurate and reliable tool to detect BCRL in the affected hand (76). Furthermore, 3D imaging has been developed to reliably characterize segmental swelling by capturing the shape information, such as circumference and circularity, which accounts for a change in volume of the upper limbs of BCRL patients (78).

LymphaTech, Inc. has developed an optical three- 
A

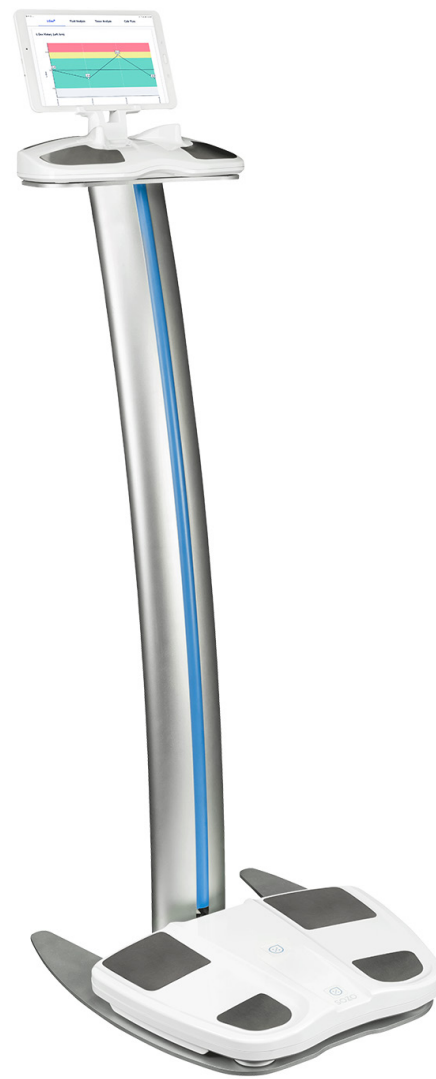

B

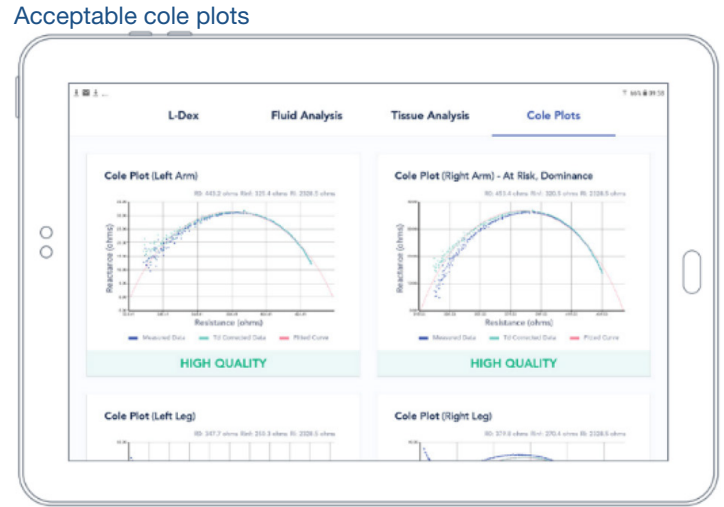

Unacceptable cole plots

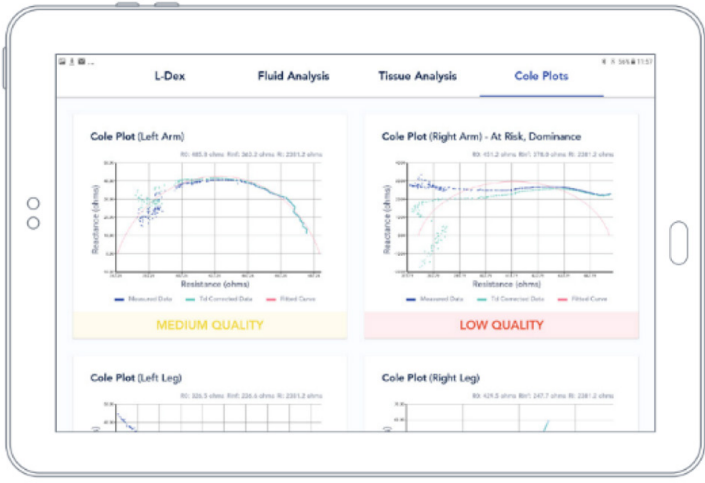

Figure 3 Use of BIS to detect BCRL. (A) SOZO ${ }^{\circledR}$ Digital Health Platform, ImpediMed Limited (67); (B) Analyzing Cole plots to determine the quality of BIS measurements (68).

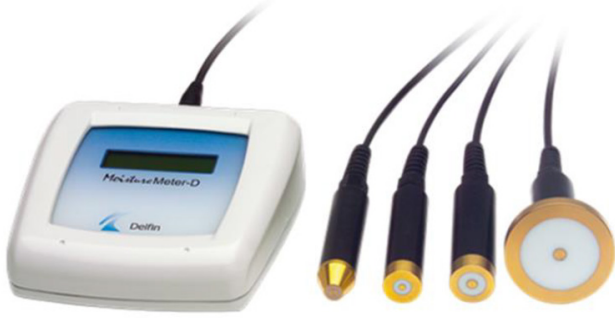

Figure 4 MoistureMeter- ${ }^{\circledR}(70)$.

dimensional imaging system (3DIS) (Figure 5), which utilizes an infrared depth sensor integrated into a computer tablet with custom accelerometer software to detect and monitor lymphedema (77). The protocol developed by Yahathugoda et al. was used to detect lymphatic filariasis (LF) of the lower limbs. Patients screened for LF stand at the center of an open space with their feet shoulder-width apart. Standing 2-3 feet away from the patient, the examiner points the tablet camera at the patient's legs, adjusts the sizing box superimposed on the tablet's screen data capture window for the depth sensor, and walks in a circle surrounding the patient to capture the entire surface of both limbs, measured two times by three different examiners (77). The median intra-examiner coefficient of variation for 3 DIS volume measurement was $1.1 \%$ (IQR $0.5-2.1 \%$ ), whereas the median inter-examiner coefficient of variation (at $24 \mathrm{~cm}$ ) was $1.3 \%$ (IQR 0.8-1.9\%). Variation increased with progression of LF (77). However, in a validation study conducted by LymphaTech, Inc., LymphaTech was found to be highly correlated with water displacement and CTM $\left(r^{2}>0.98\right)$, suggesting that the device may serve as a valid tool in diagnosing BCRL (77). With adequate space, this protocol may be adapted to measure upper limbs in the detection and management of BCRL. As its role in lymphedema screening is evolving, we have incorporated LymphaTech as a research tool in MGH's screening program to validate the results of 3DIS in comparison to perometry and to define its potential adjunct role in BCRL screening. 3DIS technology, although still costly, may propose a cheaper alternative to current 

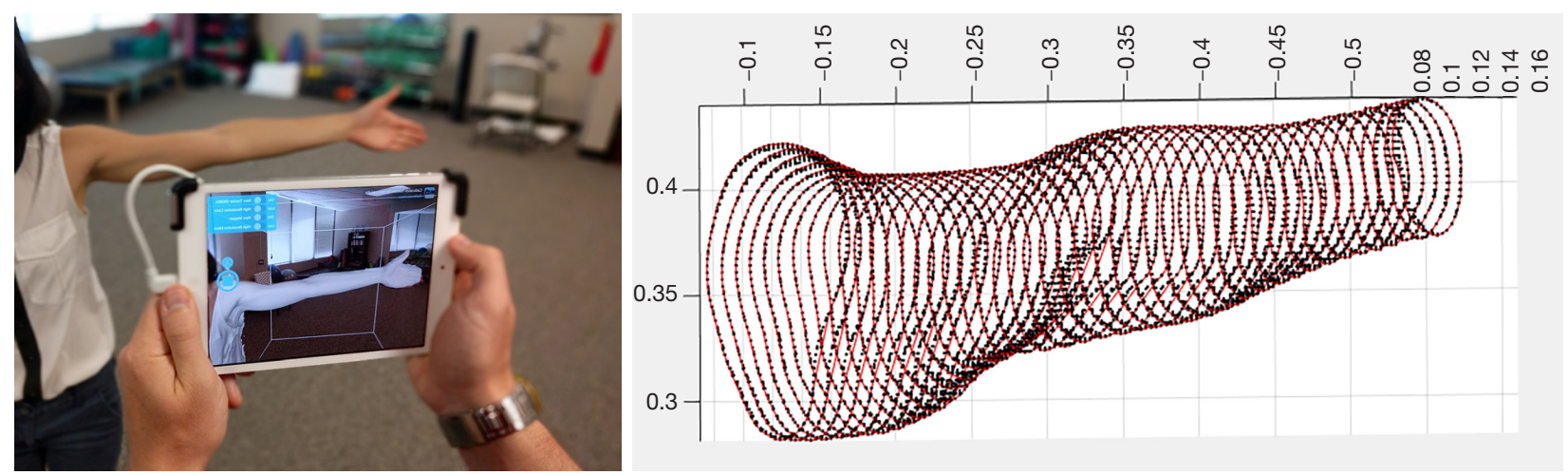

Figure 5 LymphaTech 3DIS (79).

clinical technology for calculating limb volume. Additionally, the flexibility and portability of 3DIS has broad implications for its utility in BCRL screening programs in underresourced or at-home settings.

\section{BCRL screening program at Massachusetts General Hospital (MGH)}

With increasing longevity of patients treated for BC, it is important to address potential adverse effects of treatment that impair functionality and QOL. Therefore, it is imperative to implement BCRL screening to detect and manage BCRL before progression to later stages $(32,37)$. Since its inception in 2005, the MGH prospective screening program has screened over 5,000 women to promote the early detection of BCRL. Our screening program incorporates longitudinal measures of limb volume via perometry, symptoms assessment, and clinical exam by a certified lymphedema therapist (CLT) as needed. We have implemented a multidisciplinary, team-based approach including medical, surgical, and radiation oncologists, CLTs, and nurse practitioners. The breast center is located within one outpatient ambulatory care unit, where the multidisciplinary team of medical, surgical, and radiation oncologists all see the patient. This centralized approach has been crucial to our ability to screen and follow up regularly with a large volume of patients without disrupting clinic workflow. After treatment, patients are screened every 3-8 months (3). Within our patient cohort, the median time of onset of BCRL is 18-24 months (3); however, patients are at a life-long risk of developing BCRL. Therefore, patients are screened according to their individual risk factors, symptoms and requests of physicians and CLTs, as well as the patient.
In general, BCRL is diagnosed at MGH in at-risk patients with at a relative or weight-adjusted volume change [RVC (80) or WAC (81)] of $\geq 10 \%$, whilst integrating patient symptoms and confirming through clinical exam by a CLT. BCRL is not ruled out at RVC or WAC $<10 \%$, but rather volume data is integrated with individual risk factors, patient-reported symptoms and clinical exam by a CLT.

When suspected upon limb volume measurement or patient report of symptoms, patients are referred to the CLT by their provider for evaluation. Patients are seen in a timely fashion, and diagnosis and treatment are considered. BCRL is staged at MGH using the ISL staging system (82). When patients are diagnosed with BCRL, treatment may include some or all of the following: patient education, exercise, compression, manual lymphatic drainage and self-care.

\section{Limb volume measurement: infrared perometry}

At MGH it is the role of Clinical Research Coordinators (CRCs) to operate the Perometer and collect limb measurements; however, this role could be assumed by anyone who is trained and works within close proximity to the Perometer, e.g., a medical assistant, nurse, resident, etc. (3). All measurements are taken $53 \mathrm{~mm}$ from the fingertips (Perometer default) to the axillary crease while the patient is seated perpendicular to the adjustable table and shoulder is abducted $90^{\circ}$ from the midline (Figure 1). Both the table and chair can be adjusted to achieve the ideal position. Measurements are performed in triplicate on each arm (3).

\section{Quantifying arm volume change}

As previously discussed, a baseline measurement is crucial 
for accurate diagnosis (51). We developed two formulae that incorporate preoperative baseline measures and calculate relative change. The RVC equation, developed in 2008, quantifies arm volume changes in unilaterally affected patients. In summary, $\mathrm{RVC}=\left(\mathrm{A}_{2} / \mathrm{U}_{2}\right) /\left(\mathrm{A}_{1} / \mathrm{U}_{1}\right)-1$, where $A_{1}$ and $A_{2}$ are arm volumes on the affected side at pre- and postoperative measurements and $U_{1}$ and $U_{2}$ are arm volumes on the contra-lateral side at corresponding time points (48). In 2013, our team developed the weight-adjusted change (WAC) equation to be used for bilaterally affected patients, which analyzes the baseline preoperative measurement and current median arm volume to calculate a change in independent arm volumes $(81)$. Briefly, WAC $=\left(\mathrm{A}_{2} \mathrm{~W}_{1}\right) /$ $\left(W_{2} A_{1}\right)-1$, where $A_{1}$ and $A_{2}$ are pre- and postoperative arm volumes, respectively, and $W_{1}$ and $W_{2}$ are the patient's weights at the corresponding time points (81).

Segmental analysis is calculated on arm volume measurements upon request by the patient's CLT and/or if a patient note that symptoms are localized to a specific region of the arm. Segmental measurements are performed on the PeroPlus $2000^{\circledR}$ software. First, anatomical landmarks are identified and marked [wrist, olecranon process $(\mathrm{OP}), \pm 10 \mathrm{~cm}$ from the $\mathrm{OP}$ marker, axilla] such that volumes from each arm are analyzed in triplicate of the following segments: full arm, wrist to axilla, OP to axilla, wrist to $\mathrm{OP}, \mathrm{OP} \pm 10 \mathrm{~cm}$. In a retrospective subset analysis of a larger prospective cohort trial, Stout et al. noted a significant volume increase $(\mathrm{P}<0.001)$ at the arm segment between $20-30 \mathrm{~cm}$ before a diagnosis of subclinical BCRL (83). Segmental volume changes at the 20-30 $\mathrm{cm}$ and $10-20 \mathrm{~cm}$ segment correlated to the total limb volume change with coefficients of determination of $r^{2}=0.952$ and $r^{2}=0.845$, respectively (83). In a cohort of 55 unilaterally-affected BCRL patients, Daniell et al. found that more than $1 / 3(19 / 55)$ of patients with an overall RVC of $5 \%$ to $<10 \%$ had at least one segment with an RVC $\geq 10 \%$ (84). Segmental increases in volume may be predicted on multivariate analysis by history of ALND with $(\mathrm{P}=0.018)$ or without RLNR $(\mathrm{P}=0.069)$ and a $\mathrm{BMI}$ $\geq 30 \mathrm{~kg} / \mathrm{m}^{2}$ at baseline $(\mathrm{P}=0.076)(84)$. Segmental increases in volume may be predictive of BCRL, so we perform segmental analysis of upper limb volumes selectively, using the PeroPlus $2000^{\circledR}$ software and REDCap in conjunction with symptom assessment, to help identify areas of swelling when whole arm volume does not indicate BCRL. Our implementation of segmental analysis is currently evolving as we establish its utility in BCRL screening.

We realize that at times it is not possible to obtain preoperative baseline limb volume measurements, or patients may come to the facility to transfer care from an outside hospital without baseline limb volume measurements. In these cases, as with all of our patients, we rely on integration of screening data, including limb volume measurements at the time of follow-up [this would be absolute arm volume difference left to right, realizing that many patients have baseline arm asymmetry (51)], subjective or symptom data and patient report, and clinical examination. We consider individualized risk factors, and synthesize this data together when considering a BCRL diagnosis for each patient (3).

\section{Epic $^{\circledR}$}

Epic $^{\circledR}$ is an electronic medical record software accessible by all providers within the Partners HealthCare System. This software is used across academic research centers and community health locations alike. It also has a patient portal component, Partners Patient Gateway, which allows patients to access medical records and communicate with providers (85). One can access pathology notes and other relevant provider notes to gain information such as the patient's diagnosis date, age at diagnosis, previous $\mathrm{BC}$ diagnoses and breast surgeries, as well as course of treatment to identify potential risk factors for developing lymphedema. One can also access vital signs, height and weight, and body mass index, which are used in BCRL analysis. Furthermore, Epic $^{\circledR}$ is used to determine other clinical drug and/or radiation trials on which patients are enrolled, which may put them at higher risk for edema. Determination of patient risk, based on the factors listed above, informs patient education and guides the frequency of patient follow-up appointments. Within Epic ${ }^{\circledR}$, the CRC documents a patient's arm volume measurements. All patients seen in the clinic are documented in Epic ${ }^{\circledR}$, such that all longitudinal measurements are viewable. These measurements can be accessed by anyone with access to the patient's chart, notably CLTs and oncology providers to track the patient's progress and use this information to guide further treatment.

\section{REDCap}

Data collected by the Lymphedema Research Program is maintained in REDCap, a research database tool used for secure data collection (86). Our REDCap database captures information on patient demographics, clinical 
and pathophysiological factors, treatment-related data, lymphedema therapy treatment, symptoms, and risk factors. Limb volume measurements are transcribed from the PeroPlus $2000^{\circledR}$ software into REDCap for all patients with a pre-surgical baseline to calculate the RVC of the affected arm for unilaterally affected patients and a WAC for bilaterally affected patients. For patients with a $\mathrm{SOZO}^{\circledR}$ baseline, these measurements are also transcribed into REDCap for tracking of L-Dex changes. REDCap houses the data of patients with a baseline measurement and is also used to calculate segmental measurements. Moreover, The Lymphedema Research Program at MGH utilizes R-Studio to clean data for analysis in Microsoft Excel. Utilization of REDCap, R-Studio and Microsoft Excel have allowed a focus on research database management and thereby facilitated the speed of analysis within our research program.

\section{Electronic collection of symptoms}

Patients with BCRL report lower QOL than those without BCRL (29) and the symptoms experienced by BCRL patients may account for some of these impairments (49). The role of symptoms, such as heaviness, fullness, and/or numbness of the affected limb (87), has been of increasing focus in the literature, as there is a nonlinear relationship between symptoms and severity of BCRL $(29,88)$. Moreover, not one symptom or group of symptoms alone is diagnostic for BCRL. It is important that BCRL screening not only incorporates objective measurements and clinical examination when necessary, but also incorporates symptoms (87). Development of symptoms may precede detectable swelling and may indicate early BCRL $(49,87)$.

Our program aims to not only assess symptoms but also the associated distress caused by the symptoms, as BCRLassociated symptoms may cause social, psychological, and/or emotional distress $(29,89)$. For these reasons, the Lymphedema Research Program has implemented the BC and Lymphedema Symptom Experience Index (BCLESEI), established by Fu et al. $(30,90)$. The BCLE-SEI is a 5-point Likert-type self-report instrument evaluating the occurrence and distress from real-time BCRL symptoms (90). The survey assesses 26 different symptoms in the following manner (91): "Have you had _ ? If YES, How severe?" Patients can rate each symptom on a scale of 0-4: 0 indicating no presence of symptoms and 4 indicating very severe symptoms (90). Additionally, the BCLE-SEI evaluates distress experienced in activities of daily living, affect, cognitive, and behavioral distress (90). To assess distress caused by BCRL symptoms, the survey asks, for example: "How much do your symptoms negatively affect your mood or cause psychological distress?" It is also validated in both paper and electronic forms (91). We chose to implement the BCLE-SEI, a reliable and valid tool for patients with BCRL to report symptoms, as this survey aptly captures distress associated with BCRL symptoms.

At MGH, the BCLE-SEI is administered via an electronic tablet with an individualized $\mathrm{QR}$ code such that responses are automatically downloaded to REDCap. A secure, HIPAA-compliant link may also be shared with the patient via email to complete the survey with automatic database filling remotely on a personal smart phone or computer. If the patient prefers, a paper BCLE-SEI can be administered in place of the tablet. When administering paper surveys, they are labeled with the patient's unique identifier and date given, and patients are provided with a self-addressed and pre-stamped envelope for ease of entering these responses into REDCap retrospectively. Symptoms are recorded in REDCap so they may be incorporated into data analysis for each research project.

\section{Case-Study: following a patient through use of technology in the MGH BCRL screening program}

The following hypothetical case study will be used to highlight the use of technology on a patient in the MGH's BCRL prospective screening program.

On August 26, the CRC makes a schedule for BCRL screening for August 27. In Epic ${ }^{\circledR}$, the CRC has access to all patient appointments in the unit. It is the role of the CRC to crosscheck all patients with appointments in Epic ${ }^{\circledR}$ for August 27 with the PeroPlus $2000^{\circledR}$ software for history of BCRL screening. All newly diagnosed preoperative and postoperative patients, as well as those being seen by a CLT, and those with identified risk factors who have a baseline measurement are considered for addition to the schedule. The CRC finds, upon consulting the notes within the PeroPlus $2000^{\circledR}$ software, that Jane Doe is a patient who has received a right lumpectomy and an ALND with 4/23 nodes positive for metastasis in April 18, 2017 and has received RLNR. Mrs. Doe was last measured on October 6, 2018 and is coming in for a follow-up appointment with her radiation oncologist tomorrow.

Due to the presence of multiple risk factors for BCRL, Ms. Doe would be considered a high priority patient for 
a screening visit. The CRC utilizes Ambulatory Patient Tracking, an electronic system that allows providers to monitor the status of patients within the unit throughout their care, so that he or she can collect Ms. Doe either from the waiting room, or after her appointment from her room within the Breast Center. If Ms. Doe has either been told by her physician that she should receive arm measurements or feels symptomatic, she can notify the front desk, who in turn pages the CRC to alert him or her that the patient requests measurements so that the patient can be seen promptly.

During the appointment, the CRC measures both of her arms in triplicate with the Perometer. Throughout these measurements, the CRC asks Ms. Doe about her risk factors and symptoms, recording her risk factors as well as her arm volumes calculated by the Perometer (Figure 2) into REDCap/EPIC ${ }^{\circledR}$. Once the CRC has measured Ms. Doe's arms and input these values into REDCap/EPIC ${ }^{\circledR}$ to calculate her right RVC, Ms. Doe agrees to complete the BCLE-SEI on the tablet. The CRC generates Ms. Doe's unique QR code through REDCap and enters it into REDCap on the tablet to give Ms. Doe access to the survey. While Ms. Doe completes the survey, the CRC inputs the measurement data from REDCap to a flow sheet within Epic $^{\circledR}$ so that today's measurement, along with Ms. Doe's longitudinal series of follow-up measurements are visible in her synopsis. Once Ms. Doe has completed the survey, the CRC notifies her that she has a right RVC of $7.65 \%$. We have found it prudent to report the patient's RVC to her after completing the symptoms questionnaire, to not unconsciously bias her answers.

If not already referred to a CLT, the CRC educates the patient that she may be referred to a CLT for further treatment if she wishes, as she has a slightly elevated RVC, which means she is at risk of progression to BCRL (44). If Ms. Doe requests a referral, the CRC sends an email to the CLT, who practices in a separate wing of the hospital. The CLT then sets up the referral for BCRL evaluation and treatment at the earliest available opening. If Ms. Doe had a right $\mathrm{RVC}$ of $10 \%$ or greater, she would be referred to a CLT automatically. However, even without an RVC $\geq 10 \%$, unique risk factors of the patient and symptoms reported, in addition to the calculated RVC would be considered by the team in making the decision to refer a patient to a CLT. Once established with a CLT, Ms. Doe may come in for regular arm measurements, which may be scheduled within Epic $^{\circledR}$. If Ms. Doe chooses to forgo treatment with a CLT, the CRC may make a note in the PeroPlus $2000^{\circledR}$ software that Ms. Doe had an elevated right RVC of $7.65 \%$ on $8 / 27 / 19$, and to measure her at her following appointment, such that when the CRC is making the schedule, it will be clear that the patient is of high priority.

The geographically-centralized patient care team and multidisciplinary approach to patient-centered care has been of the utmost importance in facilitating longitudinal screening using rather basic technology, without disrupting clinic work flow for the BCRL Screening Program at MGH.

\section{Future directions}

The future of BCRL screening and management lies in valid, reliable, diagnostically accurate, portable, and affordable tools for screening in the clinic and for patient self-screening in the community $(5,32,33,37,92)$. Although the centralized geographic location of our care team has been instrumental to the success of our patient-centered continuum of care at $\mathrm{MGH}$, we recognize that this is not always feasible, particularly in community hospitals and under-resourced settings. Although this paper does focus on the role of technology in sustaining a comprehensive prospective screening program for BRCL, we want to underscore the importance of screening, with or without technology. We want to emphasize the importance of implementing a screening program wherein baseline measurements are taken and follow-up measurements are performed accurately and consistently. CTM is a reliable and valid form of volume-based measurement $(32,33,82,92)$. for BCRL screening and it may be used in conjunction with commercially available software programs that calculate limb volumes from girth measurements (57).

There are many new technologies being developed to improve surveillance of BCRL. Machine learning may take on a crucial role in future clinical care, as many risk factors for BCRL have been described; however, it is difficult to predict a patient's unique risk that incorporates the importance of individual risk factors (93-95). By analyzing 30 variables, ranging from demographics, surgery, radiation, and systemic therapy, Taghian et al. sought to analyze all potential risk factors to generate a user-friendly nomogram to generate a weighted and specific prediction of a patient's risk of BCRL (94). We see machine learning to be a useful tool not only to generate a patient-specific prediction of risk of developing BCRL, but also to discover the underlying relationship between BCRL and its relevant symptoms, which are difficult to analyze with classical statistics (91). 
A study performed by $\mathrm{Fu}$ et al. analyzed the reliability and accuracy of five algorithms for machine learning and detection of BCRL based on symptoms (91). Although Fu et al. could not yet verify patients' BCRL status, their study shows that by using an artificial neuronal network (ANN) classifier, the accuracy, sensitivity, and specificity of realtime BCRL detection can be improved (91).

Furthermore, investing in telehealth and provision of a tool that is both portable and affordable could revolutionize screening and management of BCRL. 3D imaging technology (75-78), made available through an augmented mobile device, supplemented with high-quality, patient-centered educational material, could help bridge gaps in the continuum of care $(90,96)$. In a 2016 study, Fu et al. described the development of The-Optimal-LymphFlow health IT system (TOLF), a patient-centered mobile interface focused on education and self-care habits, that can collect self-reported symptoms data via the BCLESEI with high reliability and validity $(90,96)$. We hope to see the development of technology that facilitates at-home measurement that does not compromise the reliability, validity, and accuracy of BCRL detection and management, but rather provides a more comprehensive continuum of care and improved QOL.

In conclusion, prospective lymphedema screening for patients treated for BC is recommended. In concordance with guidelines published by the NLN, ISL, ASBrS, and NCCN, we advise the implementation of comprehensive screening programs, utilizing baseline measurements, as standard of care to facilitate the early detection of BCRL $(5,32,33,37)$. However, in the absence of an established program, patient education regarding individual risk factors, potential BCRL symptoms and the imperative nature of early diagnosis should be used (90).

\section{Acknowledgments}

Funding: The project was supported by Award Number R01CA139118 (AG Taghian) and Award Number P50CA08393 (AG Taghian) from the National Cancer Institute. The content is solely the responsibility of the authors and does not necessarily represent the official views of the National Cancer Institute or the National Institutes of Health. This program is supported by the Adele McKinnon Research Fund for Breast Cancer-Related Lymphedema, the Heinz Family Foundation, and the Olayan-Xefos Family Fund for Breast Cancer Research.

\section{Footnote}

Provenance and Peer Review: This article was commissioned by the Guest Editor (Mei R. Fu) for the series "Real-Time Detection and Management of Chronic Illnesses" published in $m$ Health. The article was sent for external peer review organized by the Guest Editor and the editorial office.

Conflicts of Interest: All authors have completed the ICMJE uniform disclosure form (http://dx. doi. org/10.21037/ mhealth-19-218). The series "Real-Time Detection and Management of Chronic Illnesses" was commissioned by the editorial office without any funding or sponsorship. CLB reports grants from the National Cancer Institute, funding from the Adele McKinnon Research Fund for Breast Cancer-Related Lymphedema, the Heinz Family Foundation, and from Olayan-Xefos Family Fund for Breast Cancer Research, during the conduct of the study; personal fees from PureTech Health, outside the submitted work. AGT reports grants from National Cancer Institute, funding from the Adele McKinnon Research Fund for Breast Cancer-Related Lymphedema, the Heinz Family Foundation, and the Olayan-Xefos Family Fund for Breast Cancer Research, during the conduct of the study; personal fees from PureTech Health, equipment loaned from Impedimed, outside the submitted work. The authors have no other conflicts of interest to declare.

Ethical Statement: The authors are accountable for all aspects of the work in ensuring that questions related to the accuracy or integrity of any part of the work are appropriately investigated and resolved.

Open Access Statement: This is an Open Access article distributed in accordance with the Creative Commons Attribution-NonCommercial-NoDerivs 4.0 International License (CC BY-NC-ND 4.0), which permits the noncommercial replication and distribution of the article with the strict proviso that no changes or edits are made and the original work is properly cited (including links to both the formal publication through the relevant DOI and the license). See: https://creativecommons.org/licenses/by-nc-nd/4.0/.

\section{References}

1. American Cancer Society Cancer Statistics Center. How common is breast cancer? Available online: https://www. google.com/amp/s/amp.cancer.org/cancer/breast-cancer/ 
about/how-common-is-breast-cancer.html

2. DiSipio T, Rye S, Newman B, et al. Incidence of unilateral arm lymphoedema after breast cancer: A systematic review and meta-analysis. Lancet Oncol 2013;14:500-15.

3. Brunelle C, Skolny M, Ferguson C, et al. Establishing and sustaining a prospective screening program for breast cancer-related lymphedema at the Massachusetts General Hospital: Lessons Learned. J Pers Med 2015;5:153-64.

4. Lawenda BD, Mondry TE, Johnstone PAS. Lymphedema: A primer on the identification and management of a chronic condition in oncologic treatment. CA Cancer J Clin 2009;59:8-24.

5. ISL. The diagnosis and treatment of peripheral lymphedema: 2016 consensus document of the International Society of Lymphology. Lymphology 2016;49:170-84.

6. Warren LEG, Miller CL, Horick N, et al. The impact of radiation therapy on the risk of lymphedema after treatment for breast cancer: A prospective cohort study. Int J Radiat Oncol Biol Phys 2014;88:565-71.

7. Ridner SH, Dietrich MS, Stewart BR, et al. Body mass index and breast cancer treatment-related lymphedema. Support Care Cancer 2011;19:853-7.

8. Jammallo LS, Miller CL, Singer M, et al. Impact of body mass index and weight fluctuation on lymphedema risk in patients treated for breast cancer. Breast Cancer Res Treat 2013;142:59-67.

9. Kilbreath SL, Refshauge KM, Beith JM, et al. Risk factors for lymphoedema in women with breast cancer: A large prospective cohort. Breast 2016;28:29-36.

10. Tsai RJ, Dennis LK, Lynch CF, et al. The risk of developing arm lymphedema among breast cancer survivors: A meta-analysis of treatment factors. Ann Surg Oncol 2009;16:1959-72.

11. McLaughlin SA, Wright MJ, Morris KT, et al. Prevalence of lymphedema in women with breast cancer 5 years after sentinel lymph node biopsy or axillary dissection: Objective measurements. J Clin Oncol 2008;26:5213-9.

12. Miller CL, Colwell AS, Horick N, et al. Immediate implant reconstruction is associated with a reduced risk of lymphedema compared to mastectomy alone: A prospective cohort study. Ann Surg 2016;263:399-405.

13. Crosby MA, Card A, Liu J, et al. Immediate breast reconstruction and lymphedema incidence. Plast Reconstr Surg 2012;129:789e-95e.

14. Card A, Crosby M, Liu J, et al. Reduced incidence of breast cancer-related lymphedema following mastectomy and breast reconstruction versus mastectomy alone. Plast
Reconstr Surg 2012;130:1169-78.

15. Lee KT, Mun GH, Lim SY, et al. The impact of immediate breast reconstruction on post-mastectomy lymphedema in patients undergoing modified radical mastectomy. The Breast 2013;22:53-7.

16. Siotos C, Sebai ME, Wan EL, et al. Breast reconstruction and risk of arm lymphedema development: A metaanalysis. J Plast Reconstr Aesthet Surg 2018;71:807-18.

17. Armer JM, Ballman KV, McCall L, et al. Factors Associated With Lymphedema in Women With NodePositive Breast Cancer Treated With Neoadjuvant Chemotherapy and Axillary Dissection. JAMA Surg 2019;154:800

18. Zhu W, Li D, Li X, et al. Association between adjuvant docetaxel-based chemotherapy and breast cancer-related lymphedema. Anticancer Drugs 2017;28:350-5.

19. Ohsumi S, Shimozuma K, Ohashi Y, et al. Subjective and objective assessment of edema during adjuvant chemotherapy for breast cancer using taxane-containing regimens in a randomized controlled trial: The national surgical adjuvant study of breast cancer 02 . Oncology 2012;82:131-8.

20. Invernizzi M, Michelotti A, Noale M, et al. Breast Cancer Systemic Treatments and Upper Limb Lymphedema: A Risk Assessment Platform Encompassing Tumor-Specific Pathological Features Reveals the Potential Role of Trastuzumab. JCM 2019;8:138.

21. Swaroop MN, Ferguson CM, Horick NK, et al. Impact of adjuvant taxane-based chemotherapy on development of breast cancer-related lymphedema: Results from a large prospective cohort. Breast Cancer Res Treat 2015;151:393-403.

22. Ganju RG, Savvides G, Korentager S, et al. Incidence of breast lymphedema and predictors of its development in patients receiving whole breast radiation therapy after breast-conservation surgery. Lymphology 2019;52:126-33.

23. Newman B, Lose F, Kedda MA, et al. Possible genetic predisposition to lymphedema after breast cancer. Lymphat Res Biol 2012;10:2-13.

24. Visser J, Van Geel M, Cornelissen AJM, et al. Breast Cancer-Related Lymphedema and Genetic Predisposition: A Systematic Review of the Literature. Lymphat Res Biol 2019;17:288-93.

25. Fu MR. Breast cancer-related lymphedema: Symptoms, diagnosis, risk reduction, and management. World J Clin Oncol 2014;5:241-7.

26. Taghian NR, Miller CL, Jammallo LS, et al. Lymphedema following breast cancer treatment and impact on quality of 
life: A review. Crit Rev Oncol Hematol 2014;92:227-34.

27. O'Toole JA, Ferguson CM, Swaroop MN, et al. The impact of breast cancer-related lymphedema on the ability to perform upper extremity activities of daily living. Breast Cancer Res Treat 2015;150:381-8.

28. Armer JM, Ballman KV, McCall L, et al. Lymphedema symptoms and limb measurement changes in breast cancer survivors treated with neoadjuvant chemotherapy and axillary dissection: results of American College of Surgeons Oncology Group (ACOSOG) Z1071 (Alliance) substudy. Support Care Cancer 2019;27:495-503.

29. Ridner SH. Quality of life and a symptom cluster associated with breast cancer treatment-related lymphedema. Support Care Cancer 2005;13:904-11.

30. Fu MR, McDaniel RW, Rhodes VA. Measuring symptom occurrence and symptom distress: Development of the symptom experience index. J Adv Nurs 2007;59:623-34.

31. Chachaj A, Malyszczak K, Pyszel K, et al. Physical and psychological impairments of women with upper limb lymphedema following breast cancer treatment. Psychooncology 2010;19:299-305.

32. NLN Medical Advisory Committee. Screening And Early Detection Of Breast Cancer-Related Lymphedema: The Imperative. National Lymphedema Network 2011. Available online: https://lymphnet.org/position-papers

33. McLaughlin SA, Staley AC, Vicini F, et al. Considerations for clinicians in the diagnosis, prevention, and treatment of breast cancer-related lymphedema: Recommendations from a multidisciplinary expert ASBrS panel: Part 1: Definitions, Assessments, Education, and Future Directions. Ann Surg Oncol 2017;24:2818-26.

34. Ochalek K, Gradalski T, Partsch H. Preventing Early Postoperative Arm Swelling and Lymphedema Manifestation by Compression Sleeves After Axillary Lymph Node Interventions in Breast Cancer Patients: A Randomized Controlled Trial. J Pain Symptom Manage 2017;54:346-54.

35. Stout Gergich NL, Pfalzer LA, McGarvey C, Springer $\mathrm{B}$, et al. Preoperative assessment enables the early diagnosis and successful treatment of lymphedema. Cancer 2008;112:2809-19.

36. Koelmeyer LA, Borotkanics RJ, Alcorso J, et al. Early surveillance is associated with less incidence and severity of breast cancer-related lymphedema compared with a traditional referral model of care. Cancer 2019;125:854-62.

37. National Comprehensive Cancer Network. Survivorship (Version 1.2020). Available online: https://www.nccn.org/ professionals/physician_gls/pdf/survivorship.pdf

38. McLaughlin SA, Staley AC, Vicini F, et al. Considerations for Clinicians in the Diagnosis, Prevention, and Treatment of Breast Cancer-Related Lymphedema, Recommendations from an Expert Panel: Part 2: Preventive and Therapeutic Options. Ann Surg Oncol 2017;24:2827-35.

39. Ridner SH, Dietrich MS, Cowher MS, et al. A Randomized Trial Evaluating Bioimpedance Spectroscopy Versus Tape Measurement for the Prevention of Lymphedema Following Treatment for Breast Cancer: Interim Analysis. Ann Surg Oncol.2019;26:3250-9.

40. Kaufman DI, Shah C, Vicini FA, et al. Utilization of bioimpedance spectroscopy in the prevention of chronic breast cancer-related lymphedema. Breast Cancer Res Treat 2017;166:809-15.

41. Akita S, Nakamura R, Yamamoto N, et al. Early detection of lymphatic disorder and treatment for lymphedema following breast cancer. Plast Reconstr Surg 2016;138:192e-202e.

42. Shah C, Arthur DW, Wazer D, et al. The impact of early detection and intervention of breast cancerrelated lymphedema: A systematic review. Cancer Med 2016;5:1154-62.

43. Soran A, Menekse E, Girgis M, et al. Breast cancer-related lymphedema after axillary lymph node dissection: Does early postoperative prediction model work? Support Care Cancer 2016;24:1413-9.

44. Specht MC, Miller CL, Russell TA, et al. Defining a threshold for intervention in breast cancer-related lymphedema: What level of arm volume increase predicts progression? Breast Cancer Res Treat 2013;140:485-94.

45. Lacomba MT, Sánchez MJY, Goñi ÁZ, et al. Effectiveness of early physiotherapy to prevent lymphoedema after surgery for breast cancer: Randomised, single blinded, clinical trial. BMJ 2010;340:140.

46. Box RC, Reul-Hirche HM, Bullock-Saxton JE, et al. Physiotherapy after breast cancer surgery: results of a randomised controlled study to minimise lymphoedema. Breast Cancer Res Treat 2002;75:51-64.

47. O’Toole J, Jammallo LS, Miller CL, et al. Screening for breast cancer-related lymphedema: The need for standardization. Oncologist 2013;18:350-2.

48. Ancukiewicz M, Miller CL, Skolny MN, et al. Comparison of relative versus absolute arm size change as criteria for quantifying breast cancer-related lymphedema: The flaws in current studies and need for universal methodology. Breast Cancer Res Treat 2012;135:145-52.

49. Armer JM, Stewart BR. A comparison of four diagnostic 
criteria for lymphedema in a post-breast cancer population. Lymphat Res Biol 2005;3:208-17.

50. Centers NAP for B. National Accreditation Program For Breast Centers Standards Manual. Chicago, IL: American College of Surgeons, 2017.

51. Sun F, Skolny MN, Swaroop MN, et al. The need for preoperative baseline arm measurement to accurately quantify breast cancer-related lymphedema. Breast Cancer Res Treat 2016;157:229-40.

52. Lee M-J, Boland RA, Czerniec S, et al. Reliability and Concurrent Validity of the Perometer for Measuring Hand Volume in Women With and Without Lymphedema. Lymphat Res Biol 2011;9:13-8.

53. Stanton AWB, Northfield JW, Holroyd B, et al. Validation of optoelectornic limb volumeter. Lymphology 1997;30:77-97.

54. Sun F, Hall A, Tighe MP, et al. Perometry versus simulated circumferential tape measurement for the detection of breast cancer-related lymphedema. Breast Cancer Res Treat 2018;172:83-91.

55. Pero-System Messgeräte. Perometer 350 NT. Available online: http://pero-system.de/en/perometer-350-nt/

56. Smoot BJ, Wong JF, Dodd MJ. Comparison of diagnostic accuracy of clinical measures of breast cancerrelated lymphedema: Area under the curve. Arch Phys Med Rehabil 2011;92:603-10.

57. Professional LV. Limb Volumes Professional 2019. Available online: http://www.clinsoft.org/

58. York SL, Ward LC, Czerniec S, et al. Single frequency versus bioimpedance spectroscopy for the assessment of lymphedema. Breast Cancer Res Treat 2009;117:177-82.

59. Moseley A, Piller N. Reliability of bioimpedance spectroscopy and tonometry after breast conserving cancer treatment. Lymphat Res Biol 2008;6:85-7.

60. Cornish B, Chapman M, Hirst C, et al. Early diagnosis of lymphedema using multiple frequency bioimpedance. Lymphology 2001;34:2-11.

61. Qin ES, Bowen MJ, Chen W. Diagnostic accuracy of bioimpedance spectroscopy in patients with lymphedema: A retrospective cohort analysis. J Plast Reconstr Aesthet Surg 2018;71:1041-50.

62. Barrio AV, Eaton A, Frazier TG. A Prospective Validation Study of Bioimpedance with Volume Displacement in Early-Stage Breast Cancer Patients at Risk for Lymphedema. Ann Surg Oncol 2015;22:370-5.

63. Seward C, Skolny M, Brunelle C, et al. A comprehensive review of bioimpedance spectroscopy as a diagnostic tool for the detection and measurement of breast cancer-related lymphedema. J Surg Oncol 2016;114:537-42.

64. Fu MR, Cleland CM, Guth AA, et al. L-Dex Ratio in Detecting Breast Cancer-Related Lymphedema: Reliability, Sensitivity, and Specificity. Lymphology 2013;46:85-96.

65. Ridner SH, Dietrich MS, Spotanski K, et al. A prospective study of L-Dex values in breast cancer patients pretreatment and through 12 months postoperatively. Lymphat Res Biol 2018;16:435-41.

66. Temkin SM, Mcguire KP, Bellavance EC, et al. Preventing Breast cancer-related lymphedema in high-risk Patients: The impact of a structured surveillance Protocol Using Bioimpedance spectroscopy. Front Oncol 2018;8:12.

67. ImpediMed Inc. SOZO® Digital Health Platform 2019. Available online: www.impedimed.com

68. ImpediMed. SOZO ® Measurement Guide 2019;56. Available online: http://impedimed.com/wp-content/ uploads/2019/07/LBL-504-US-Rev-D-SOZOMeasurement-Guide.pdf

69. ImpediMed. Cole Plot Analysis 2019:1-7. Available online: http://impedimed.com/wp-content/uploads/2019/07/ LBL-504-US-Rev-D-SOZO-Measurement-Guide.pdf

70. Delfin Technologies. MoistureMeterD 2009. Available online: http://www.delfintech.com/en/product_ information/moisturemeterd/

71. Bakar Y, Tuğral A, Üyetürk Ü. Measurement of Local Tissue Water in Patients with Breast Cancer-Related Lymphedema. Lymphat Res Biol 2018;16:160-4.

72. Mayrovitz HN. Assessing Free and Bound Water in Skin at $300 \mathrm{MHz}$ using TDC measurements with the Moisture MeterD. In: Greene AK, Brorson H, Slavin SA, editors. Lymphedema: Presentation, Diagnosis, and Treatment. Springer International Publishing, 2015:133-48.

73. Mayrovitz HN, Weingrad DN, Davey S, et al. Local Tissue Water in At-Risk and Contralateral Forearms of Women with and without Breast Cancer TreatmentRelated Lymphedema. Lymphat Res Biol 2009;7:153-8.

74. Mayrovitz HN. Assessing local tissue edema in postmastectomy lymphedema. Lymphology 2007;40:87-94.

75. Hameeteman M, Verhulst AC, Vreeken RD, et al. 3D stereophotogrammetry in upper-extremity lymphedema: An accurate diagnostic method. J Plast Reconstr Aesthet Surg 2016;69:241-7.

76. Hoevenaren IA, Verhulst AC, Hameeteman M, et al. Three-dimensional stereophotogrammetry as an accurate tool for analyzing lymphedema of the hand. JPRAS Open 2016;10:40-6.

77. Yahathugoda C, Weiler MJ, Rao R, et al. Use of a Novel 
Portable Three-Dimensional Imaging System to Measure Limb Volume and Circumference in Patients with Filarial Lymphedema. Am J Trop Med Hyg 2017;97:1836-42.

78. Karakashian K, Shaban L, Pike C, et al. Investigation of shape with patients suffering from unilateral lymphoedema. Ann Biomed Eng 2018;46:108-21.

79. LymphaTech. What is LymphaTech? 2017. Available online: https://lymphatechnology.com/

80. Ancukiewicz M, Russell TA, Otoole J, et al. Standardized method for quantification of developing lymphedema in patients treated for breast cancer. Int J Radiat Oncol Biol Phys 2011;79:1436-43.

81. Miller CL, Specht MC, Horick N, et al. A novel, validated method to quantify breast cancer-related lymphedema (BCRL) following bilateral breast surgery. Lymphology 2013;46:64-74.

82. Lymphology TIS of. The diagnosis and treatment of peripheral lymphedema: 2016 consensus document of the International Society of Lymphology. Lymphology 2016;49:170-84.

83. Stout NL, Pfalzer LA, Levy E, et al. Segmental limb volume change as a predictor of the onset of lymphedema in women with early breast cancer. PM R 2011;3:1098-105.

84. Daniell KD, Roberts SA, Gillespie TG, et al. Assessing segmental limb volume changes for early detection of BCRL [abstract]. Chicago, IL: International Lymphedema Framework Conference, 2019.

85. Epic Systems Corporation. About Us 2019. Available online: https://www.epic.com/about

86. REDCap. ABOUT. Available online: https://projectredcap. org/about/

87. Armer JM, Radina ME, Porock D, et al. Predicting breast cancer-related lymphedema using self-reported symptoms. Nurs Res 2003;52:370-9.

88. Sierla R, Lee TSM, Black D, et al. Lymphedema following

doi: $10.21037 /$ mhealth-19-218

Cite this article as: Havens LM, Brunelle CL, Gillespie TC, Bernstein M, Bucci LK, Kassamani YW, Taghian AG. Use of technology to facilitate a prospective surveillance program for breast cancer-related lymphedema at the Massachusetts General Hospital. mHealth 2021;7:11. breast cancer: regions affected, severity of symptoms, and benefits of treatment from the patients' perspective. Clin J Oncol Nurs 2013;17:325-31.

89. Fu MR, Rosedale M. Breast cancer survivors' experiences of lymphedema-related symptoms. J Pain Symptom Manage 2009;38:849-59.

90. Fu MR, Axelrod D, Guth AA, et al. mHealth self-care interventions: Managing symptoms following breast cancer treatment. mHealth 2016;2:28-28.

91. Fu MR, Wang Y, Li C, et al. Machine learning for detection of lymphedema among breast cancer survivors. mHealth 2018;4:17-17.

92. Galiano-Castillo N, Ariza-García A, CantareroVillanueva I, et al. Agreement between telerehabilitation involving caregivers and face-to-face clinical assessment of lymphedema in breast cancer survivors. Support Care Cancer 2014;22:253-8.

93. Bevilacqua JLB, Kattan MW, Changhong Y, et al. Nomograms for predicting the risk of arm lymphedema after axillary dissection in breast cancer. Ann Surg Oncol 2012;19:2580-9.

94. Taghian A, Roberts S, Naoum G, et al. Identifying risk factors for breast cancer-related lymphedema: results from a prospective screening program. Chicago, IL: ILF Conference, 2019.

95. Gross JP, Whelan TJ, Parulekar WR, et al. Development and Validation of a Nomogram to Predict Lymphedema After Axillary Surgery and Radiation Therapy in Women With Breast Cancer From the NCIC CTG MA.20 Randomized Trial. Int J Radiat Oncol Biol Phys 2019;105:165-73.

96. Fu MR, Axelrod D, Guth A, et al. A Web- and MobileBased Intervention for Women Treated for Breast Cancer to Manage Chronic Pain and Symptoms Related to Lymphedema: Randomized Clinical Trial Rationale and Protocol. JMIR Res Protoc 2016;5:e7. 